\title{
Effects of aging on left atrial reservoir, conduit, and booster pump function: a multi-institution acoustic quantification study
}

University of Chicago, Chicago, Illinois, USA K T Spencer V Mor-Avi

L Weinert

J Bednarz

R M Lang

University of Pittsburgh, Pittsburgh, Pennsylvania, USA

J Gorcsan III

K Edelmen

University of California, San Diego, California, USA A N DeMaria

O L Kwan

Children's Hospital, Cincinnati, Ohio, USA

T R Kimball

B Glascock

King's College, London, UK

M J Monaghan

$\mathrm{J}$ Hancock

Washington University, St Louis, Missouri, USA

J E Perez

C Baumann

Correspondence to: Kirk Spencer MD, Noninvasive Cardiac Imaging Laboratory, $5841 \mathrm{~S}$ Maryland Avenue, MC5084, Chicago, IL 60637, USA kspencer@

medicine.bsd.uchicago.edu

Accepted 11 September 2000

K T Spencer, V Mor-Avi, J Gorcsan III, A N DeMaria, T R Kimball, M J Monaghan, J E Perez, L Weinert, J Bednarz, K Edelman, O L Kwan, B Glascock, J Hancock, C Baumann, R M Lang

\begin{abstract}
Objective-To assess the feasibility of measuring left atrial (LA) function with acoustic quantification (AQ) and then assess the effects of age and sex on LA reservoir, conduit, and booster pump function.

Patients and setting-165 subjects without cardiovascular disease, 3-79 years old, were enrolled by six tertiary hospital centres.

Interventions-Continuous LA AQ area data were acquired and signal averaged to form composite waveforms which were analysed off-line.

Main outcome measures-Parameters of LA performance according to age and sex.

Results-Signal averaged LA waveforms were sufficiently stable and detailed to allow automated analysis in all cases. An age related increase in LA area was noted. LA reservoir function did not vary with age or sex. All parameters of LA passive and active emptying revealed a significant age dependency. Overall, the passive emptying phase accounted for $66 \%$ of total LA emptying ranging from $76 \%$ in the youngest to $44 \%$ in the oldest decade. LA contraction accounted for $34 \%$ of atrial emptying in all subjects combined with the older subjects being more dependent on atrial booster pump function. When adjusted for atrial size, there were no sex related differences in LA function.

Conclusions-LA reservoir, conduit, and booster pump function can be assessed with automated analysis of signal averaged LA area waveforms. As LA performance varies with age, establishment of normal values should enhance the evaluation of pathologic states in which LA function is important.

(Heart 2001;85:272-277)
\end{abstract}

Keywords: aging; atrium; echocardiography

Left atrial (LA) performance is complex and includes functioning as a reservoir, conduit, and booster pump at different stages of the cardiac cycle. Abnormalities of LA performance are clinically important and found in a wide variety of common clinical conditions such as atrial fibrillation, dilated cardiomyopathy, diastolic dysfunction, and valvar heart disease. ${ }^{1-5}$ Detailed evaluation of LA function has been performed using a variety of invasive methods which combine LA pressure with volume measurement to generate pressuredimension loops. ${ }^{67}$ This approach is limited by the need for direct LA pressure recordings which precludes routine clinical use.

The most commonly used non-invasive technique to evaluate LA performance is Doppler echocardiography. However, Doppler echocardiography is limited since it provides no information about LA size and, although the use of this technique to evaluate LA booster pump function has been described, it is not suitable for the assessment of the reservoir and conduit phases of LA function. Measurement of LA size at different phases of the cardiac cycle has proven to be useful for evaluating LA performance. However, the assessment of LA volume by two dimensional (cross sectional) echocardiography requires tedious hand tracing of endocardial borders.
Acoustic quantification (AQ), an automated border detection technique, provides on-line continuous cardiac chamber area or volume over time and has been used extensively to study the left ventricle. ${ }^{8-10}$ Individual AQ waveforms can be noisy due to random and physiologic variations, but the use of signal averaging of $\mathrm{AQ}$ waveforms reduces this variation. ${ }^{11}$ Initial studies applying AQ to the LA chamber have not used signal averaging and additionally have been limited by the need to identify manually the different cardiac phases which is tedious and subjective. ${ }^{45^{12-14}} \mathrm{Al}-$ though abnormalities in disease states such as hypertension and atrial fibrillation have been demonstrated with this technique, the small number of normal subjects has limited systematic conclusions about these findings. Before evaluating LA reservoir, conduit, and booster pump function in pathologic states, a thorough understanding of LA physiology as assessed by AQ in a large group of normal subjects over a wide age range is essential.

This study sought to: (1) assess the feasibility of measuring LA reservoir, conduit, and booster pump function with computer automated analysis of signal averaged LA area waveforms; (2) assess the effects of age and sex on LA reservoir, conduit, and booster pump function; and (3) establish normal values for 


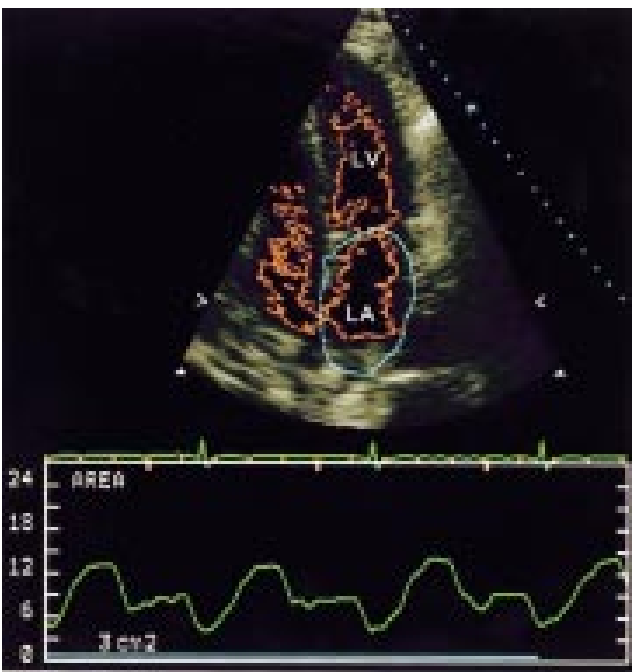

Figure 1 Apical four chamber view with acoustic quantification activated and optimised for the left atrium. The bottom of the figure shows the $L A A Q$ area waveform (green).

parameters of LA performance as determined from $A Q$.

\section{Methods}

SUBJECTS

One hundred and seventy one subjects (81 males and 90 females) aged 3-79 years old were enrolled from six participating centres. Subjects were required to have a transthoracic window adequate for performing automated border detection. Entry criteria included: (1) no clinical history of cardiovascular disease; (2) normal sinus rhythm; (3) absence of left bundle branch block; (4) normal blood pressure; (5) absence of wall motion abnormality on two dimensional echocardiographic screening; (6) no more than trivial aortic insufficiency or mild mitral, tricuspid or pulmonic regurgitation; (7) normal left ventricular mass determined using the area-length formula; (8) ejection fraction of $\geqslant 50 \%$ calculated from manual tracings of end systolic and end diastolic frames using the method of discs.

\section{ACOUSTIC QUANTIFICATION}

$\mathrm{AQ}$ is an automated online technique that analyses the ultrasound backscatter to identify the blood-tissue interface of cardiac chambers and generate area or volume waveforms within a defined region of interest. ${ }^{8-10}$ Using a SONOS 5500 (Hewlett Packard, Andover, Massachusetts, USA) images were obtained from the apical four chamber window taking care to maintain a non-foreshortened, maximally sized view of the left atrium. The automated border detection system was activated and optimised, as previously described, ${ }^{15}$ by adjusting the total gain, time gain compensation, and lateral gain. A region of interest was then drawn around the left atrium excluding the pulmonary veins. The LA area waveform was displayed and visual confirmation of consistent stable waveforms made (fig 1). Forty five seconds of LA area versus time data were acquired to magneto-optical disk. All acquisitions were made during quiet respiration without breath holding.

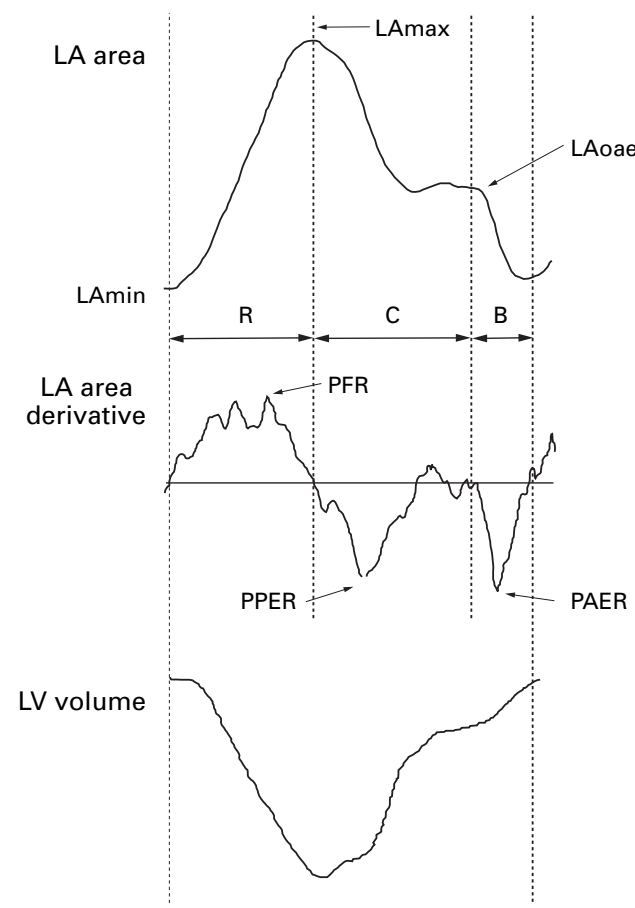

Figure 2 Signal averaged $A Q L A$ area (top) and $L A$ derivative (middle) waveforms. Left ventricular (LV) volume waveforms (bottom) are shown for reference. The phases of the cardiac cycle are labelled: $R$, reservoir; $C$, conduit; B, booster pump; LAmin, minimum area; LAmax, maximum area; LAoae, onset atrial emptying area; PFR, peak filling rate; PPER, peak passive emptying rate; PAER, peak active emptying rate.

\section{ANALYSIS}

All atrial AQ waveforms were analysed off-line using custom software. All 45 seconds of LA waveform data were signal averaged resulting in a single composite waveform. Signal averaging reduces noise and variability of the AQ waveforms. ${ }^{11}$ From this signal averaged waveform the first derivative of LA area was computed. The custom software automatically identified the phases on the LA waveform using morphologic criteria of the area and derivative curves. The minimum area (LAmin), maximum area (LAmax), and the area at the onset of atrial emptying (LAoae) were then determined (fig 2).

LA reservoir function was assessed using filling area (LAmax - LAmin), expansion index (LAmax - LAmin)/LAmin ${ }^{\star} 100$, and the peak rate of filling (PFR). The overall diastolic emptying index was calculated as (LAmax LAmin)/LAmax ${ }^{\star} 100$. LA conduit function was assessed by calculating: the passive emptying percentage of total emptying $(\mathrm{PE})=$ (LAmax - LAoae)/(LAmax - LAmin $)^{\star} 100$, passive emptying index $(\mathrm{PEI})=($ LAmax LAoae)/LAmax ${ }^{\star} 100$, and peak passive emptying rate (PPER). Parameters of atrial booster pump function included: active emptying percentage of total emptying $(\mathrm{AE})=(\mathrm{LAoae}-$ LAmin)/(LAmax - LAmin $)^{\star} 100$, active emptying index (AEI) = (LAoae - LAmin)/ LAoae 100 , and peak active emptying rate (PAER). All area derivative peak rates were expressed in absolute units $\left(\mathrm{cm}^{2} / \mathrm{s}\right)$ and normalised by the maximum atrial area (LAmax/s). 
Table 1 Normal values for $A Q L A$

\begin{tabular}{|c|c|c|c|c|c|c|c|c|c|c|c|}
\hline Decade & 1 & 2 & 3 & 4 & 5 & 6 & 7 & 8 & All & Adult & $p$ Value \\
\hline n & 16 & 21 & 27 & 2 & 24 & 16 & 18 & 18 & 165 & 128 & \\
\hline LAmin $\left(\mathrm{cm}^{2}\right)$ & $3.1(1.2)^{\star}$ & $7.0(3.3)$ & $7.5(2.7)$ & $6.8(2.9)$ & $8.1(3.9)$ & $9.3(3.0)$ & $8.2(3.4)$ & $10(3.2)^{\star}$ & $7.5(3.5)$ & $8.2(3.3)$ & $<0.0001$ \\
\hline LAmax $\left(\mathrm{cm}^{2}\right)$ & $6.6(2.2)^{\star}$ & $12(4.0)$ & $15(2.7)$ & $13(3.3)$ & $16(3.6)$ & $16(2.6)$ & $15(3.5)$ & $17(3.8)^{\star}$ & $14(4.4)$ & $15(3.6)$ & $<0.0001$ \\
\hline LAoae $\left(\mathrm{cm}^{2}\right)$ & $3.9(1.4)^{\star}$ & $8.3(3.5)$ & $9.5(2.6)$ & $8.7(3.1)$ & $10.6(4.3)$ & $12(3.2)$ & $11(3.5)$ & $14(3.2)^{\star}$ & $9.7(4.1)$ & $10.7(3.7)$ & $<0.0001$ \\
\hline $\operatorname{PFR}\left(\mathrm{cm}^{2} / \mathrm{sec}\right)$ & $19(9)^{\star}$ & $27(0)^{\star}$ & $36(10)$ & $32(7)$ & $37(13)$ & $34(10)$ & $35(12)$ & $38(12)$ & $33(12)$ & $36(11)$ & $<0.001$ \\
\hline PFR (LAmax/sec) & $2.9(0.7)$ & $2.3(0.8)$ & $2.6(0.8)$ & $2.6(0.9)$ & $2.5(0.8)$ & $2.2(0.6)$ & $2.4(0.9)$ & $2.2(0.6)$ & $2.5(0.8)$ & $2.4(0.8)$ & NS \\
\hline Filling area $\left(\mathrm{cm}^{2}\right)$ & $3.5(1.5)^{\star}$ & $5.2(1.9)^{\star}$ & $7.0(1.7)$ & $6.3(1.5)$ & $7.3(2.4)$ & $6.4(1.2)$ & $7.1(2.3)$ & $7.2(1.9)$ & $6.4(2.2)$ & $6.9(1.9)$ & $<0.001$ \\
\hline Expansion index $(\%) \dagger$ & $126(60)$ & $70(36)$ & $99(68)$ & $94(78)$ & $99(73)$ & $68(35)$ & $89(66)$ & $66(32)$ & $83(72)$ & $83(69)$ & NS \\
\hline DEI & $53(13)$ & $44(15)$ & 49 (13) & $50(14)$ & $49(15)$ & $42(10)$ & $48(16)$ & $42(10)$ & $47(14)$ & $47(13)$ & NS \\
\hline PE (\%) & $76(10)$ & $75(8)$ & $73(8)$ & $70(8)$ & $67(15)$ & $62(12)^{\star}$ & $58(13)^{\star}$ & $44(10)^{\star}$ & $66(14)$ & $64(14)$ & $<0.001$ \\
\hline $\operatorname{PPER}\left(\mathrm{cm}^{2} / \mathrm{sec}\right)$ & $30(13)^{\star}$ & $42(22)$ & 49 (12) & $43(12)$ & $46(17)$ & $39(8)$ & $40(18)$ & $27(11)^{\star}$ & $41(16)$ & $42(15)$ & $<0.001$ \\
\hline PPER (LAmax/sec) & $4.5(1.6)$ & $3.6(1.9)$ & $3.5(1.1)$ & $3.5(1.6)$ & $3.1(1.2)$ & $2.6(0.7)$ & $2.7(1.3)$ & $1.6(0.7)^{\star}$ & $3.2(1.5)$ & $2.9(1.3)$ & $<0.001$ \\
\hline PEI & $40(12)$ & $33(14)$ & $36(9)$ & $35(12)$ & $33(14)$ & $26(7)$ & $29(14)$ & $19(7)^{\star}$ & $32(13)$ & $31(12)$ & $<0.001$ \\
\hline $\mathrm{AE}(\%)$ & $24(10)$ & $25(8)$ & $27(8)$ & $30(8)$ & $33(15)$ & $38(12)^{\star}$ & $42(13)^{\star}$ & $56(10)^{\star}$ & $34(14)$ & $36(14)$ & $<0.001$ \\
\hline PAER $\left(\mathrm{cm}^{2} / \mathrm{sec}\right)$ & $15(7)^{\star}$ & $20(9)^{\star}$ & $29(12)$ & $31(12)$ & $35(17)$ & $28(10)$ & $36(13)$ & $46(12)^{\star}$ & $30(15)$ & $34(14)$ & $<0.001$ \\
\hline PAER (LAmax/sec) & $2.3(0.7)$ & $1.6(0.5)$ & $2.1(0.9)$ & $2.3(0.8)$ & $2.2(1.0)$ & $1.9(0.9)$ & $2.4(1.0)$ & $2.7(0.8)$ & $2.2(0.9)$ & $2.3(0.9)$ & 0.004 \\
\hline AEI & $21(8)$ & $17(10)$ & $22(12)$ & $24(11)$ & 25 (11) & $22(10)$ & $28(13)$ & $29(9)$ & $23(11)$ & $25(11)$ & 0.022 \\
\hline PPER/PAER & $2.2(1.0)$ & $2.4(1.6)$ & $1.9(0.7)$ & $1.7(1.2)$ & $1.6(0.8)$ & $1.5(0.9)$ & $1.2(0.6)$ & $0.6(0.3)^{\star}$ & $1.7(1.1)$ & $1.5(0.9)$ & $<0.001$ \\
\hline $\mathrm{PE} / \mathrm{AE}$ & $4.1(2.6)$ & $3.4(1.6)$ & $3.1(1.5)$ & $2.6(1.2)$ & $2.7(1.9)$ & $2.0(1.4)$ & $1.7(1.0)^{\star}$ & $0.9(0.3)^{\star}$ & $2.6(1.8)$ & $2.3(1.5)$ & $<0.001$ \\
\hline
\end{tabular}

All data presented as mean (SD) except for expansion index $\nmid$ which is presented as median (interquartile range).

${ }^{\star} \mathrm{p}<0.05$ versus decade 3 . NS, not significant.

$\mathrm{AE}$, atrial emptying fraction; AEI, atrial emptying index; AQ, acoustic quantification; DEI, diastolic emptying index; LA, left atrium; LAmax, maximum LA area; LAmin, minimum LA area; LAoae, onset atrial emptying area; PE, passive emptying fraction; PEI, passive emptying index; PAER, peak atrial emptying rate; PFR, peak filling rate; PPER, peak passive emptying rate.

Table 2 Sex differences in AQ LA parameters

\begin{tabular}{lll}
\hline & Male & Female \\
\hline LAmin $\left(\mathrm{cm}^{2}\right)$ & $7.9(3.8)$ & $7.1(3.3)$ \\
LAmax $\left(\mathrm{cm}^{2}\right)$ & $14.6(4.7)$ & $13.0(4.2)^{\star}$ \\
LAoae $\left(\mathrm{cm}^{2}\right)$ & $10.3(4.3)$ & $9.1(3.9)^{\star}$ \\
PE $(\%)$ & $71(15)$ & $70(16)$ \\
PPER $\left(\mathrm{cm}^{2} / \mathrm{sec}\right)$ & $43(17)$ & $38(16)^{\star}$ \\
AE $(\%)$ & $35(14)$ & $33(14)$ \\
PAER $\left(\mathrm{cm}^{2} / \mathrm{sec}\right)$ & $33(15)$ & $27(14)^{\star}$ \\
PPER/PAER & $1.5(0.8)$ & $1.8(1.3)$ \\
PE/AE & $2.6(1.5)$ & $2.8(1.8)$ \\
PFR $\left(\mathrm{cm}^{2} / \mathrm{sec}\right)$ & $35(12)$ & $31(12)^{\star}$
\end{tabular}

All data presented as mean (SD)

${ }^{\star} \mathrm{p}<0.05$ versus male.

See table 1 for key to abbreviations.

STATISTICAL ANALYSIS

Subjects were grouped by decades for comparison. Normally distributed variables were expressed as mean and standard deviation and non-normally distributed variables as median and interquartile range. Analysis of variance was performed to assess for age related changes in AQ parameters of LA function. Nonnormally distributed parameters were compared with a Kruskal-Wallis test. Values for male and female subjects were compared with an unpaired $t$ test. A probability value of $\mathrm{p}<0.05$ was considered significant.

\section{Results}

LA AQ data acquired in 6/171 subjects were unusable because of inadequate ECG tracings which prevented signal averaging. The age distribution of study subjects is shown in table 1 . The signal averaged LA waveforms were sufficiently stable and detailed to allow automated analysis in all cases. The normal values of all LA area AQ parameters are presented by age and sex in tables 1 and 2 .

The general morphology of the LA area waveforms revealed an increase in area during ventricular systole (reservoir phase) followed by three diastolic stages. The initial diastolic portion of the LA area waveform was noted to have a rapid decline in area (conduit phase) followed by a period of relative stable area (diastasis) and, finally, a second rapid area decline occurring during atrial contraction (booster pump phase). The LA derivative waveform demonstrated peaks during the reservoir, passive, and booster pump phases. The diastolic phase of the LA area derivative curve has a morphology similar to that of Doppler mitral inflow, as the rate of change in LA size is proportional to transmitral blood flow.

ATRIAL SIZE

The average values for LA size are shown in table 1. Age related differences in LA area were noted. The youngest subjects had smaller LA minimum, maximum, and onset atrial emptying areas. From the third to the seventh decade no significant change in LA areas were noted; however, subjects in the oldest decade had increased LA sizes. This increase in LA area in the eighth decade was not accounted for by differences in body surface area. Male subjects had statistically larger LAmax and LAoae compared to female subjects (table 2). However, after accounting for differences in body surface area, there were no sex related differences in indexed LA areas.

\section{LEFT ATRIAL FUNCTION}

LA reservoir function, assessed by the area change during LA filling and the peak rate of atrial filling, was reduced in the first two decades of life and then remained unchanged during adult life (table 1). However, when the peak rate of atrial filling was normalised for LA size by dividing by the maximum area, no age related changes in LA reservoir function were noted. Overall, the LA expanded $83 \%$ during ventricular filling with a wide spread in this parameter (range 30-560\%) caused by the variation in minimum LA areas. The systolic expansion index did not vary with age. Male subjects had larger area changes and peak rates of filling that were rendered insignificant when the larger male LA areas were controlled for.

No age related difference in the overall diastolic emptying index was noted. When diastolic LA emptying was divided into passive and active components all $\mathrm{AQ}$ parameters revealed a significant age dependency (table 1). These changes are visually apparent when 

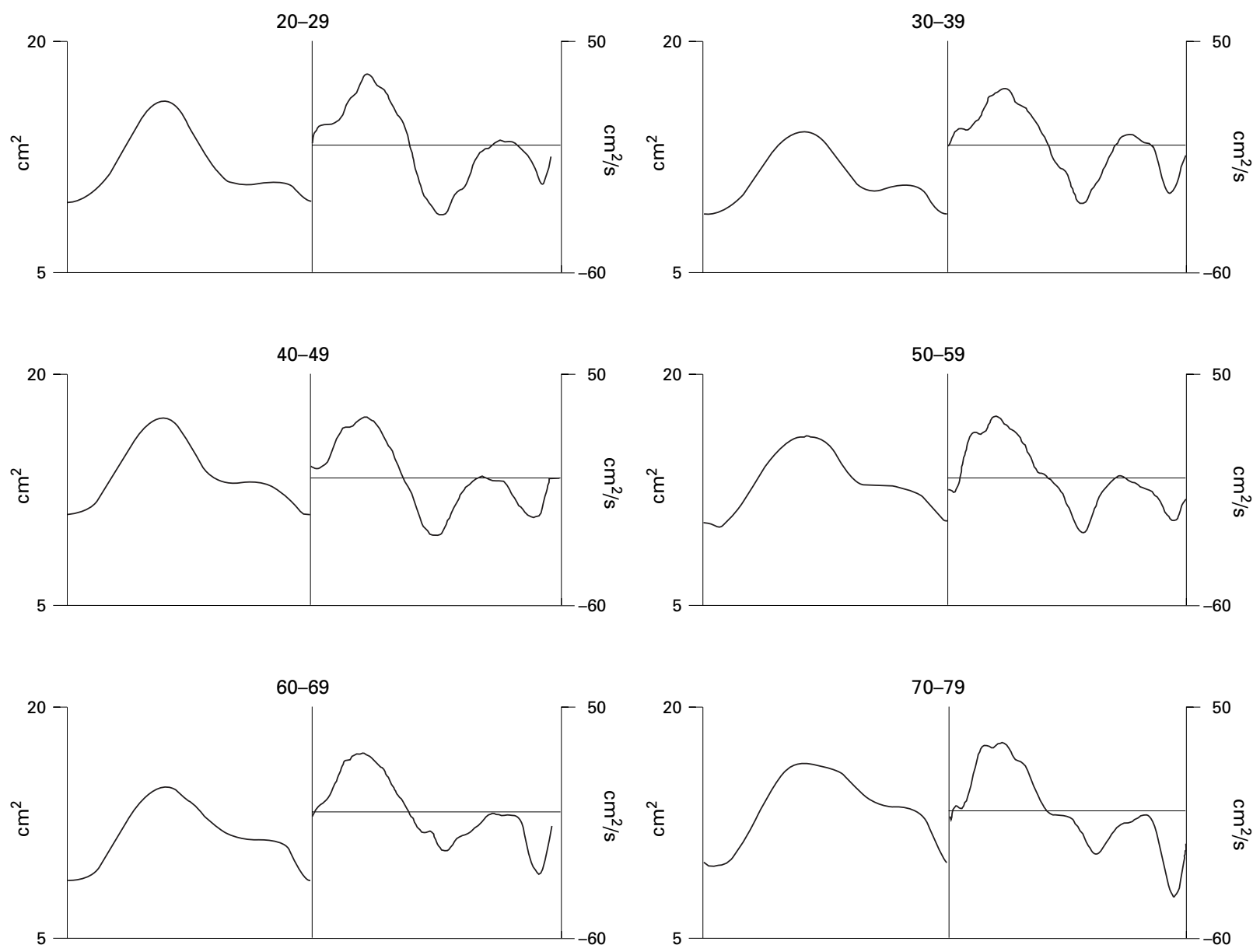

Figure 3 Composite LA waveforms generated by time normalising and averaging 10 subjects from each decade. There is an obvious morphologic change in the LA area and derivative waveform from the third to the eighth decade of life. Note the progressive decline in passive emptying and augmented active atrial booster pump function with age.

viewing composite LA waveforms generated for each decade of adult life (fig 3). The age related changes in AQ parameters of LA diastolic emptying persisted after accounting for age related changes in heart rate.

Overall, the passive phase accounted for $66 \%$ of total LA emptying. The contribution toward left ventricular (LV) filling of passive LA emptying was significantly decreased in the older three decades, reaching a low of $44 \%$ in subjects in the eighth decade (fig 4). Passive LA emptying appeared reduced in the youngest and oldest decades when assessed by the peak rate of passive emptying. However, after normalisation for LA size, this difference persisted only for the oldest subjects. LA passive function was also reduced in the oldest decade studied when measured as the passive emptying index. When adjusted for atrial size,

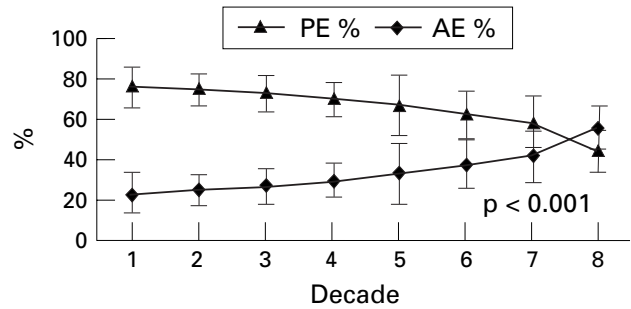

Figure 4 Age related changes in the passive and active contributions to total LA emptying in normal subjects. there are no sex related differences in LA passive function.

LA contraction accounted for $34 \%$ of atrial emptying in all subjects combined. The older three decades were more dependent on the atrial booster pump to complete LV filling compared to younger subjects as demonstrated by a larger contribution of atrial contraction to overall atrial emptying (fig 3). The peak rate of atrial emptying was reduced in the youngest subjects, but this was eliminated after correction for their smaller atrial areas. The peak atrial emptying rate and atrial emptying index were dependent on age, but when grouped by decades there were no differences compared to the third decade of life. When adjusted for atrial size, there were no sex related differences in LA booster pump function.

\section{Discussion}

ASSESSMENT OF LEFT ATRIAL FUNCTION

Although sophisticated techniques have been used to assess LA function, ${ }^{16}{ }^{16}$ these methods require invasive determination of LA pressure to generate pressure-dimension loops. Several modalities such as nuclear scintigraphy and angiography have been used to assess LA performance by measuring changes in LA volume over time. ${ }^{17} 18$ These techniques are limited by their expense, low temporal resolution, 
geometric assumptions, the need for contrast or radiopharmaceutical agents, and cumbersome acquisition. Hand drawn LA areas from two dimensional echocardiographic images are easier to acquire and have demonstrated abnormalities of LA function in patients with hypertension and dilated cardiomyopathy. ${ }^{219-22}$ Manually traced two dimensional echocardiographic LA areas are limited by poor temporal resolution (typically $2-3$ area values per cardiac cycle) and tracing of LA endocardial borders on a frame-frame basis is labour intensive and tedious.

Doppler echocardiography has also been used to evaluate LA function. Several reports have suggested using pulmonary venous systolic flow as an indicator of LA reservoir function. ${ }^{23}{ }^{24}$ The peak velocity of mitral inflow during atrial contraction together with the atrial filling fraction have been used as non-invasive markers of LA booster pump function. ${ }^{25}{ }^{26}$ These mitral inflow Doppler indices, however, provide no information on atrial size or reservoir and conduit function. Pulsed wave Doppler can be combined with mitral orifice area to compute LA ejection force $\mathrm{e}^{27}$; however, this parameter likewise only assesses LA active contractile function.

AQ provides LA area measurements throughout the cardiac cycle and, unlike other techniques, can be used to assess noninvasively LA size as well as the reservoir, conduit, and booster pump function of this chamber. The LA border determined with the AQ algorithm has fine irregularities; these average out, however, to provide an accurate estimation of LA area compared to hand drawn measurements. ${ }^{72}{ }^{14}$ Although AQ of the LA has previously been combined with LA pressure measurements to assess LA function, ${ }^{371328}$ this remains primarily a research technique.

LA AQ has been used to evaluate LA performance in atrial fibrillation, postcardiac transplant, and other cardiac pathologic states. $^{4512} 14$ In addition, LA AQ has shown promise as an adjunct to Doppler echocardiography for the assessment of diastolic dysfunction in hypertensive patients. ${ }^{29}$ However, these early studies have been limited by the small number of subjects and lack of systematic comparison of all components of LA performance with a normal age matched reference population. In addition, these initial reports are hindered by the use of single LA waveforms, which demonstrate beat to beat variability, as well as the need for subjective manual analysis of these waveforms.

This study showed that continuous LA area waveforms could be signal averaged to reduce noise and variability, allowing automated detection and detailed analysis of the different phases of LA function. These signal averaged area waveforms also provide more reliable area derivative waveforms which provide additional parameters of LA performance. LA reservoir function, which reflects the ability of the LA to fill during ventricular systole, has been difficult to assess non-invasively. AQ allowed calculation of several parameters of LA reservoir function. The expansion index of $83 \%$ is simi- lar to the previously reported values of $81 \%$ and $99 \%$ in smaller studies. ${ }^{512}$ Atrial conduit and booster pump function likewise were also quantitatively assessed with several automatically computed parameters. On average, this study demonstrated that $66 \%$ of atrial emptying occurs during the passive phase whereas $34 \%$ occurs during atrial contraction. The peak rate of emptying during the conduit phase is higher than that of the booster pump phase, and averages 1.7 times that of peak active emptying.

\section{AGE DEPENDENCY OF LEFT ATRIAL SIZE AND} FUNCTION

An age related increase in LA size has been noted previously using two dimensional echocardiography. ${ }^{20}$ Wang and colleagues, however, did not note an increase in LA size with age ${ }^{30}$ but that study only included subjects under age 65 , which is consistent with our results demonstrating no change in atrial size until the eighth decade. No prior reports have assessed the age related changes of all aspects of atrial performance in a large group of normal subjects. These age related changes are qualitatively apparent when inspecting the LA area waveforms from the different decades of life (fig 3 ). This study provides normal values of LA AQ parameters, which quantify these physiologic changes that occur in the LA area waveforms over age.

The use of AQ allowed calculation of several parameters reflecting the reservoir function of the LA. These parameters remained stable over the six decades of adult life evaluated in this study. The preserved LA expansion during the reservoir phase is important to maintain adequate LV filling. As the mitral valve is closed during ventricular systole, LA pressure is determined by both the magnitude of LA filling and atrial compliance. By maintaining LA filling, the early diastolic atrioventricular gradient required to fill the $\mathrm{LV}$ during passive filling is preserved. In addition, preserved atrial filling helps maintain the LA size at the onset of atrial contraction (atrial preload) which also augments LV filling.

In contrast to atrial filling, the characteristics of atrial emptying change with age. This study demonstrated a decline in LA passive emptying with age in normal subjects. This likely represents an age related change in LV properties leading to diastolic dysfunction which impairs passive atrial emptying during early diastole. However, there is an increase in atrial booster pump function with age which compensates for the decrease in early diastolic filling. This finding has been previously recognised using Doppler echocardiography. ${ }^{31-33}$ The observed augmentation in LA contractile function may be in part mediated via a Starling effect, as this study has demonstrated atrial size at the onset of LA contraction (atrial preload) is larger in older subjects. LA size at the onset of atrial contraction is a major determinant of LA ejection force. ${ }^{20}$ The passive and active emptying indices of $32 \%$ and $23 \%$ are different than previously reported $(25 \% \text { and } 26 \%)^{5}$ but this is likely due to the older age range in that study (31-63 years old). 
LIMITATIONS

Not all subjects can be evaluated with this technique since automated border detection, similar to other ultrasound based imaging techniques, requires an adequate two dimensional image. However, in our experience $85 \%$ of subjects have adequate windows to obtain LA AQ waveforms. The patients studied did not represent a true random selection of the general population. Although the analysis in this study was performed off-line, the signal averaging and automated analysis have been recently incorporated into a prototype ultrasound system (HP SONOS 5500) to provide on-line evaluation of LA performance.

\section{CONCLUSIONS}

The results of this study, obtained in a large group of normal subjects, demonstrated that LA reservoir, conduit, and booster pump function can be assessed with automated analysis of signal averaged LA area waveforms. These results also demonstrate that LA performance, as assessed by $A Q$, varies with age. This age dependency has important implications for future studies involving LA pathophysiology whether based on AQ or other modalities. The normal values established in this study should enhance the evaluation of pathologic states in which LA function is assessed with AQ.

1 Matsuda $\mathrm{Y}$, Toma Y, Ogawa $\mathrm{H}$, et al. Importance of left atrial function in patients with myocardial infarction. Circulation 1983;67:566-71.

2 Triposkiadis F, Pitsavos C, Boudoulas H, et al. Left atrial myopathy in idiopathic dilated cardiomyopathy. Am Heart ${ }^{\prime}$ 1994;128:308-15.

3 Stefanadis C, Dernellis J, Stratos C, et al. Effects of balloon mitral valvuloplasty on left atrial function in mitral stenosis as assessed by pressure-area relation. $7 \mathrm{Am}$ Coll Cardiol 1998;32:159-68.

4 Cresci S, Goldstein JA, Cardona $\mathrm{H}$, et al. Impaired left atrial function after heart transplantation: disparate contribution function after heart transplantation: disparate contribution of donor and recipient atrial components studied on-line with quantitative echocard
plantation 1995;14:647-53.

5 Feinberg MS, Waggoner AD, Kater KM, et al. Echocardiographic automatic boundary detection to measure left atrial function after the maze procedure. F Am Soc Echocardiog 1995;8:139-48.

6 Hoit BD, Shao Y, Gabel M, et al. In vivo assessment of left atrial contractile performance in normal and pathological conditions using a time-varying elastance model. Circulation 1994;89:1829-38

7 Stefanadis C, Dernellis J, Stratos C, et al. Assessment of left atrial pressure - area relation in humans by means of retrograde left atrial catheterization and echocardiographic automatic boundary detection: effects of Dobutamine. $\mathcal{F}$ Am Coll Cardiol 1998;31:426-36.

8 Waggoner AD, Miller JG, Perez JE. Two-dimensional echocardiographic automatic boundary detection for echocardiographic automatic boundary detection for evaluation of left ventricular function in unselect
patients. $7 \mathrm{Am}$ Soc Echocardiogr 1994;7:459-64.

9 Gorcsan JI, Morita S, Mandarino WA, et al. Twodimensional echocardiographic automated border detecdimensional echocardiographic automated border detec-
tion accurately reflects changes in left ventricular volume. $\mathcal{F}$ tion accurately reflects changes in left
Am Soc Echocardiogr 1993;6:482-9.

Am Soc Echocardiogr 1993;6:482-9.
10 Kimball TR, Witt SA, Daniels SR, et al. The role of automatic endocardial edge detection in the evaluation of left ventricular diastolic function in children. F $\mathrm{Am}$ Soc Echocardiogr 1996;9:18-26.
11 Mor-Avi V, Gillesberg IE, Korcarz C, et al. Improved quantification of left ventricular function by applying signal Am Soc Echocardiogr 1995;8:679-89.

12 Waggoner AD, Barzilai B, Miller JG, et al. On-line assessment of left atrial area and function by echocardiographic automatic boundary detection. Circulation 1993; 88:1142-9.

13 Stefanadis C, Dernellis J, Toutouzas P. Evaluation of the left atrial performance using acoustic quantification. Echocardiography 1999;16:117-25.

14 Clarkson PBM, Wheeldon NM, Lim PO, et al. Left atrial size and function: assessment using echocardiographic automatic boundary detection. Br Heart f 1995;74:664-70.

15 Bednarz JE, Marcus RH, Lang RM. Technical guidelines for performing automated border detection studies. $7 \mathrm{Am}$ Soc Echocardiogr 1995;8:293-305.

16 Alexander J, Sunagawa K, Chang N, et al. Instantaneous pressure-volume relation of the ejecting canine left atrium. Circulation Res 1987;61:209-19.

17 Murray JA, Kennedy JW, Figley MM. Quantitative angiocardiography. II. The normal left atrial volume in man. Circulation 1968;37:800-4.

18 Bough EW, Gandsman EJ, Shulman RS. Measurement of normal left atrial function with gated radionuclide angiography. Am $\mathcal{F}$ Cardiology 1981;48:473-8.

19 Barbier P, Alioto G, Guazzi MD. Left atrial function and ventricular filling in hypertensive patients with paroxysmal atrial fibrillation. $7 \mathrm{Am}$ Coll Cardiol 1994;24:165-70.

20 Triposkiadis F, Tenolouris K, Androulakis A, et al. Left atrial mechanical function in the healthy elderly: new insights from a combined assessment of changes in atrial volume and transmitral flow velocity. $\mathcal{F} \mathrm{Am}$ Soc Echocardiogr 1995;8:801-9.

21 Triposkiadis F, Trikas A, Pitsavos C, et al. Relation of exercise capacity in dilated cardiomyopathy to left atrial size and systolic function. Am 7 Cardiol 1992;70:825-7.

22 Dernellis JM, Stefanadis CI, Zacharoulis AA, et al. Left atrial mechanical adaptation to long-standing hemodynamic loads based on pressure-volume relations. Am 7 Cardiol 1998;81:1138-43.

23 Nagano R, Masuyama T, Naka M, et al. Contribution of atrial reservoir function to ventricular filling in hypertensive patients. Effects of nifedipine administration. Hypertension 1995;26:815-9.

24 Oki T, Tabata T, Yamada H, et al. Assessment of abnormal left atrial relaxation by transesophageal pulsed Doppler echocardiography of pulmonary venous flow velocity. Clin Cardiol 1998;21:753-8.

25 Oki T, Fukuda N, Iuchi A, et al. Left atrial systolic performance in the presence of elevated left ventricular enddiastolic pressure: evaluation by transesophageal pulsed Doppler echocardiography of left ventricular inflow and pulmonary venous flow velocities. Echocardiography 1996; 13:23-32.

26 Manning WJ, Leeman DE, Gotch PJ, et al. Pulsed Doppler evaluation of atrial mechanical function after electrical cardioversion of atrial fibrillation. 7 Am Coll Cardiol 1989;13: 617-23.

27 Manning WJ, Silverman DI, Katz S, et al. Atrial ejection force: a noninvasive assessment of atrial systolic function. $\mathcal{F}$ Am Coll Cardiol 1993:22:221-5.

28 Stefanadis C, Dernellis J, Stratos C, et al. Assessment of left atrial pressure-area relation in humans by means of retrograde left atrial catheterization and echocardiographic automatic boundary detection: effects of dobutamine. $\mathcal{f} \mathrm{Am}$ Coll Cardiol 1998;31:426-36.

29 Spencer KT, Mor-Avi V, Weinert L, et al. Age dependency of eft atrial and left ventricular acoustic quantification waveforms for the evaluation of diastolic performance in left ventricular hypertrophy. 7 Am Soc Echocardiogr 1998;11: 1027-35.

30 Wang Y, Gutman JM, Heilbron D, et al. Atrial volume in a normal adult population by two-dimensional echocardiography. Chest 1984;86:595-601.

31 Zuccala G, Cocchi A, Lattanzio F, et al. Effect of age on left atrial function in patients with coronary artery disease. Cardiology 1994;85:8-13

32 Kuo LC, Quinones MA, Rokey R, et al. Quantification of atrial contribution to left ventricular filling by pulsed Doppler echocardiography and the effect of age in normal and diseased hearts. Am f Cardiol 1987;59:1174-8.

33 Kitzman DW, Sheikh KH, Beere PA, et al. Age-related alterations of Doppler left ventricular filling indexes in normal subjects are independent of left ventricular mass, heart rate, contractility and loading conditions. F Am Coll Cardiol 1991;18:1243-50 